\title{
STING-associated vasculopathy with onset in infancy: a familial case series report and literature review
}

\author{
Yan Wang ${ }^{1 \#}$, Fan Wang ${ }^{1,2 \#}$, Xiaolei Zhang ${ }^{1,3,4,5}$ \\ ${ }^{1}$ Department of Pulmonary and Critical Care Medicine, Center of Respiratory Medicine, China-Japan Friendship Hospital, Beijing, China; ${ }^{2}$ The \\ Sixth Medical Center of PLA General Hospital, Beijing, China; ${ }^{3}$ Institute of Respiratory Medicine, Chinese Academy of Medical Sciences, Peking \\ Union Medical College, Beijing, China; ${ }^{4}$ Capital medical university, Beijing, China; ${ }^{5}$ Peking University Health Science Center, Beijing, China \\ \#These authors contributed equally to this work. \\ Correspondence to: Professor Xiaolei Zhang. Department of Pulmonary and Critical Care Medicine, Center of Respiratory Medicine, China-Japan \\ Friendship Hospital, 2 East Yinghua (Cherry Blossom) Road, Hepingli, Chaoyang District, Beijing 100029, China. Email: yutian728@sina.com.
}

\begin{abstract}
Stimulator of interferon genes (STING1) is a key intermediary in activating the type I IFN response. STING-associated vasculopathy with onset in infancy (SAVI) is a very rare autoinflammatory disease that is caused by heterozygous gain-of-function mutations in STING1. SAVI typically manifests as neonatal-onset systemic inflammation, interstitial lung disease (ILD), and severe cutaneous vasculopathy located in acral regions, including fingers, toes, ears, and nose. Severity of ILD and recurrent pulmonary infections are crucial for the prognosis. Therapeutic options for SAVI are quite limited, and JAK inhibitors are considered to be a promising treatment according to several recent case reports. We report on a familial case series of SAVI with the R281Q mutation in the STING1 gene with predominant ILD manifestations, absence of cutaneous lesions, and poor response to ruxolitinib. Moreover, we reviewed all the case reports of SAVI in English published in the PubMed database. The atypical phenotype of the current cases adds to the growing list of inflammatory syndromes associated with SAVI. The literature analysis suggests that the severity and natural courses of the disease seem to be independent of the mutation type. Although JAK inhibitors may be a promising treatment, the therapeutic effect for different phenotypes and disease statuses of SAVI warrants further investigation.
\end{abstract}

Keywords: STING-associated vasculopathy with onset in infancy (SAVI); Janus kinase inhibitors (JAK inhibitors); interstitial lung disease (ILD)

Submitted Aug 30, 2020. Accepted for publication Dec 18, 2020.

doi: $10.21037 / \mathrm{atm}-20-6198$

View this article at: http://dx.doi.org/10.21037/atm-20-6198

\section{Introduction}

Stimulator of interferon genes (STING), which is encoded by the gene transmembrane protein 173 (STING1), is a key intermediary in activating the type I interferon (IFN) response (1). Pathogenic heterozygous gain-of-function (GOF) variants in STING1 result in constitutive activation of STING and the clinical syndrome known as STINGassociated vasculopathy with onset in infancy (SAVI) (2). SAVI is characterized by early-onset systemic inflammation, cutaneous vasculopathy, and interstitial lung disease (ILD).
The severity of ILD and the presence of repeated lung infections determine the prognosis of the disease (3).

Therapeutic management of SAVI is challenging. It has been reported that systemic corticosteroids are partially effective in some cases; however, most patients respond poorly to disease-modifying therapies, such as corticosteroids, immunosuppressants and biologic agents (1). In addition, the prognosis of SAVI is generally poor, especially in patients with severe lung involvement with high mortality in the first two decades of life. In light of the encouraging results obtained with the use of Janus kinase (JAK) inhibitors in 


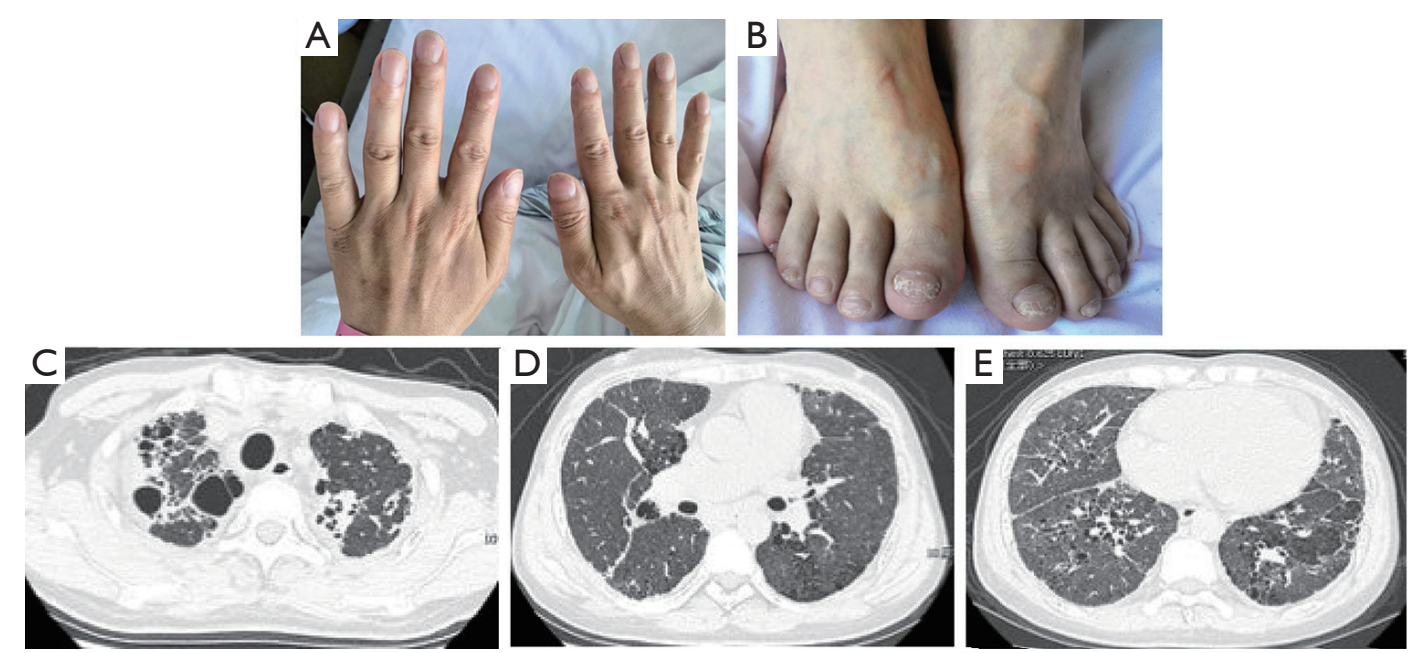

Figure 1 Clinical manifestations and chest image of case 1. (A) Clubbing fingers of case 1. (B) Nail dystrophy of toes in case 1. (C-E) Chest high-resolution computed tomography scan of case 1 . The presence of cystic airspaces in upper lobes, and bilateral reticular and groundglass opacities in the lower lobes in case 1 .

some SAVI cases $(4,5)$, this treatment is presumed to be the most promising therapy for patients with different STING1 variants. Here, we present the first familial SAVI case series with the R281Q variant with severe pulmonary involvement, in which treatment with the JAK1/2 inhibitor ruxolitinib showed poor response. We present the following cases in accordance with the CARE reporting checklist (available at http://dx.doi.org/10.21037/atm-20-6198).

\section{Case presentation}

All procedures performed in studies involving human participants were in accordance with the ethical standards of our institutional ethics committee and with the Helsinki Declaration (as revised in 2013). Written informed consent was obtained from the patient.

\section{Case 1}

A 37-year-old male presented with progressively exertional dyspnea. He had an 18-year history of abnormal imaging on chest radiograph. Four months prior, he was admitted to the local hospital due to severe cough, expectoration, dyspnea (NYHA III), nausea and vomiting. He was diagnosed as ILD accompanied by pneumonia and received antibiotics (penicillin and levofloxacin, 2 weeks) and systemic corticosteroids (methylprednisolone $40 \mathrm{mg}$, 4 weeks) without symptom improvement. Clubbing fingers and nail dystrophy were evident (Figure $1 A, B$ ). No rashes, livedo reticularis or telangiectasias were observed.

Laboratory tests found an elevated C-reactive protein (CRP) level $(5.53 \mathrm{mg} / \mathrm{dL}$, reference range: $<0.8 \mathrm{mg} / \mathrm{dL})$. The autoimmune workup was positive for antinuclear antibody, anti-dsDNA antibody, anti-cyclic citrullinated peptide (CCP) antibody, anti-Jo-1 antibody and IgG antibody to phospholipids (49; reference range, <20). Immunological testing revealed elevated levels of IgA $(494 \mathrm{mg} / \mathrm{dL})$ and positive for rheumatoid factor (RF). Increased CD8+ T lymphocytes counts and normal CD4+ T lymphocytes counts were identified. High-resolution CT (HRCT) scanning of the chest exhibited bilateral ground-glass and reticular opacities as well as cystic changes (Figure 1C,D,E). The arterial blood gases analysis (breathing room air) revealed hypoxemia [partial pressure of oxygen $\left(\mathrm{pO}_{2}\right) 65 \mathrm{mmHg}$ ] and partial pressure of carbon dioxide $\left(\mathrm{pCO}_{2}\right)$ of $33.9 \mathrm{mmHg}$. Pulmonary function tests showed a severe restrictive pattern [total lung capacity (TLC); $47.5 \%$ of predicted] with a decreased diffusing capacity for carbon monoxide (DLCO) (22.4\% of predicted). The echocardiogram demonstrated signs of right ventricle pressure overload and estimated significantly elevated systolic pulmonary pressure $(84 \mathrm{mmHg})$.

\section{Case 2}

Both sons of our index case suffered from recurrent dyspnea, cough and wheezing associated with exertion after two 


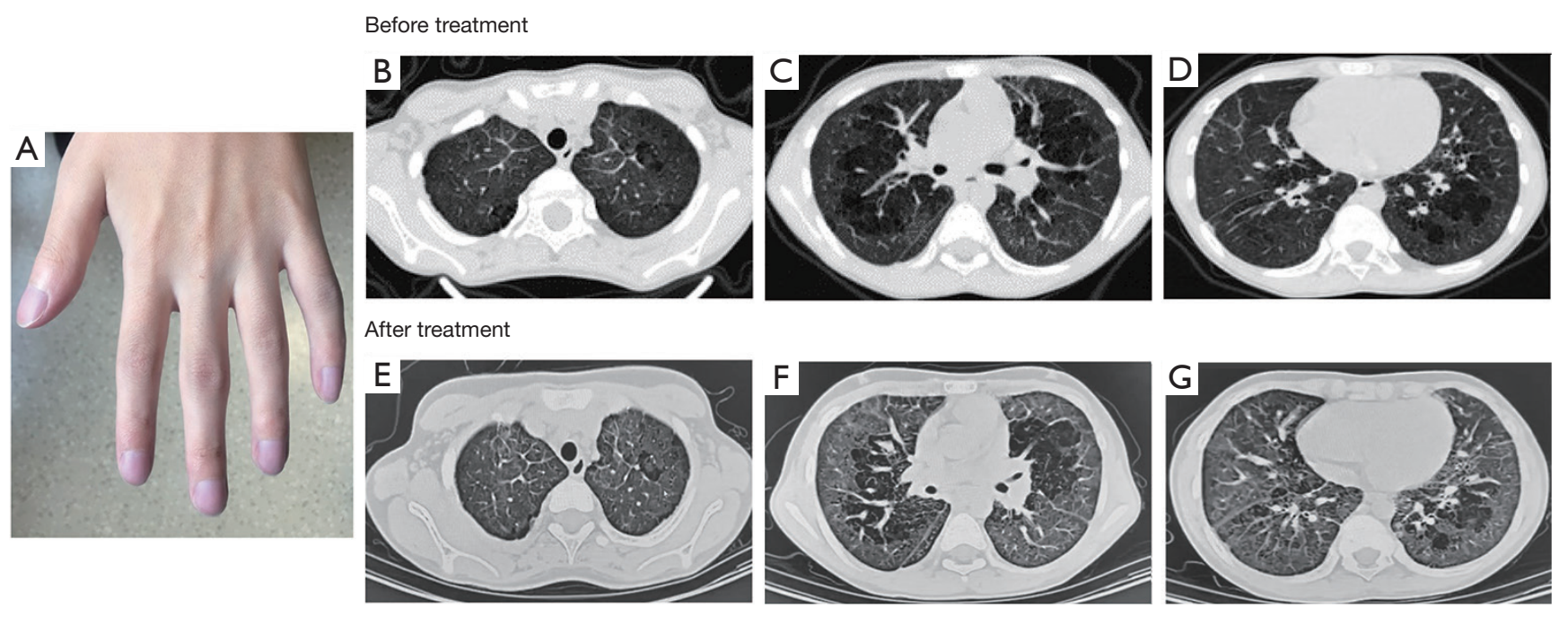

Figure 2 Clinical manifestations and chest image of case 2. (A) Clubbing fingers of case 2. (B-G) Chest high-resolution computed tomography scan of case 2. Diffused ground-glass opacities and focal lung hyperinflation in case 2.

years of age. Case 2, a 13-year-old son, displayed retarded growth with a height and weight below the 3 rd percentiles for age (131.5 cm and $31.5 \mathrm{~kg}$, respectively). He exhibited finger clubbing and suffered from recurrent migratory polyarthritis involving the interphalangeal, wrist, knee and ankle joints with impaired motion of the wrists (Figure $2 A$ ). Elevated antinuclear antibody titer $(1: 1,280)$ were detected. Pulmonary function tests exhibited a restrictive pattern with TLC $61.5 \%$ and DLCO $48.8 \%$ predicted. HRCT revealed diffuse ground-glass opacities (Figure $2 B, C, D$ ). After the treatment of ruxolitinib (JAK1/2 inhibitor), HRCT of the chest was shown in Figure 2E,F, G.

\section{Case 3}

The six-year-old son (case 3) also had failure to thrive with a height of $114 \mathrm{~cm}$ (between the 3rd and 10th percentiles) and weight of $16.25 \mathrm{~kg}$ (below the $3 \mathrm{rd}$ percentile). He did not have skin lesions or symptoms of arthritis. Clubbing fingers were observed (Figure $3 A$ ). The antinuclear antibody titer was 1:320. Diffused ground-glass and fine reticular opacities were demonstrated on HRCT of the chest (Figure $3 B, C, D$ ). He did not receive any treatment. After one year of follow-up, HRCT of the chest did not show obvious difference (Figure 3E,F,G).

Genetic analysis of the three cases by Sanger sequencing demonstrated a heterozygous STING1 (NM_198282) variant c. $841 \mathrm{G}>\mathrm{A}$, p.Arg $281 \mathrm{Gln}$, p.R281Q, which has previously been described to cause an autosomal dominant form of SAVI (6). Genetic testing of the parents of case 1 revealed that the pathogenic variant had occurred de novo. All 3 cases were heterozygous carriers of this variant (Figure $4 A, B$ ). IFN proteins and the levels of circulating inflammatory cytokines, which were detected via ELISA (Human IFN 9-Plex ELISA Kit, PBL Assay Science, USA), were significantly increased in these cases compared to controls (Figure 5). The control group consisted of 8 healthy adult men with an average age of 42 years. The three cases received the diagnose of SAVI.

Due to low platelet levels $\left(<100 \times 10^{9} / \mathrm{L}\right)$, case 1 and case 2 underwent treatment with ruxolitinib (JAK1 $/ 2$ inhibitor $)$ at doses of $5 \mathrm{mg}(0.20 \mathrm{mg} / \mathrm{kg} / \mathrm{d})$ and $2.5 \mathrm{mg}$ $(0.16 \mathrm{mg} / \mathrm{kg} / \mathrm{d})$, respectively, twice per day. Increased dosing of ruxolitinib was poorly tolerated due to unusual bleeding or bruising. Case 3 did not take ruxolitinib. Case 1 died four months later due to progressive ILD and heart failure. After one year of therapy, polyarthritis and arthralgia in case 2 were not ameliorated. The interstitial abnormalities on HRCT scan revealed no improvement for case 2 (Figure $2 E, F, G$ ). The antinuclear antibody titer remained high in case 2 and case 3 (1:320 and 1:160, respectively). IFN proteins as well as circulating inflammatory cytokines did not improve (Figure 5).

\section{Literature review}

Among all the English literature on the PubMed database 


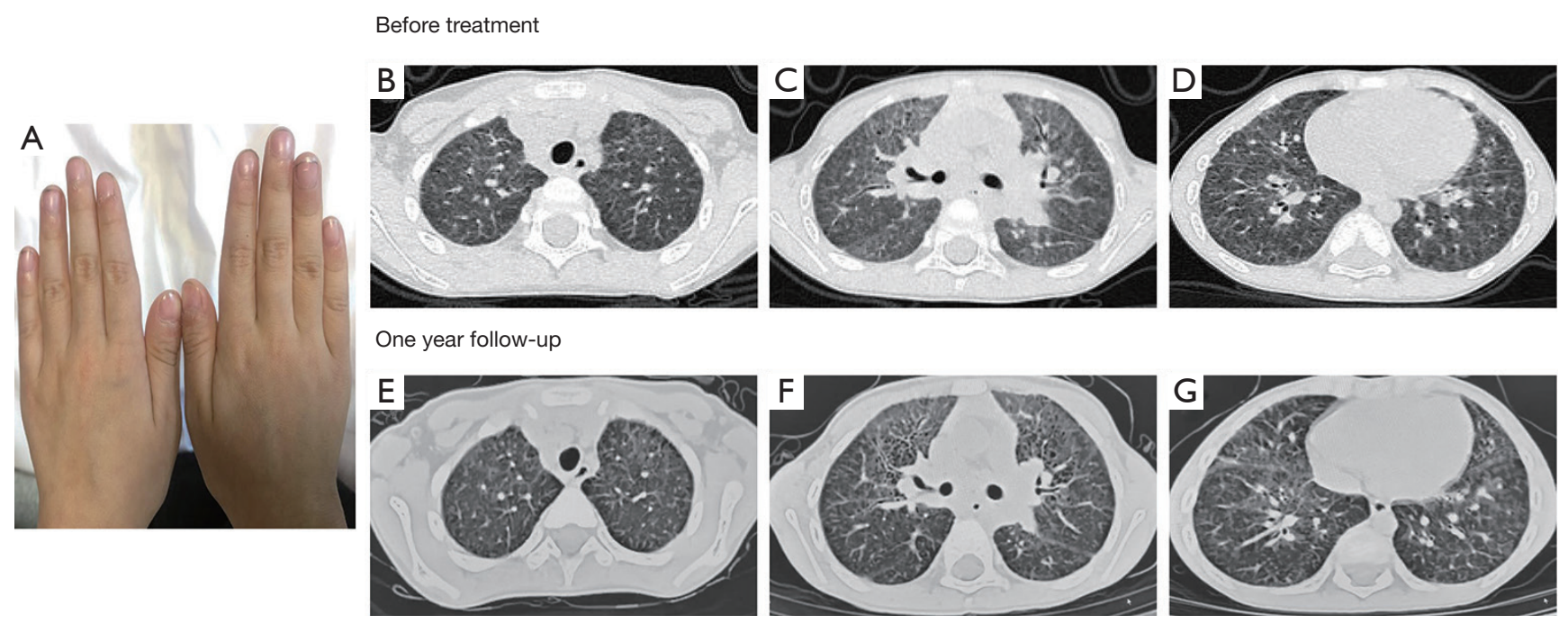

Figure 3 Clinical manifestations and chest image of case 3. (A) Clubbing fingers of case 3. (B-G) Chest high-resolution computed tomography scan of case 3. Diffused ground-glass and fine reticular opacities in case 3 .

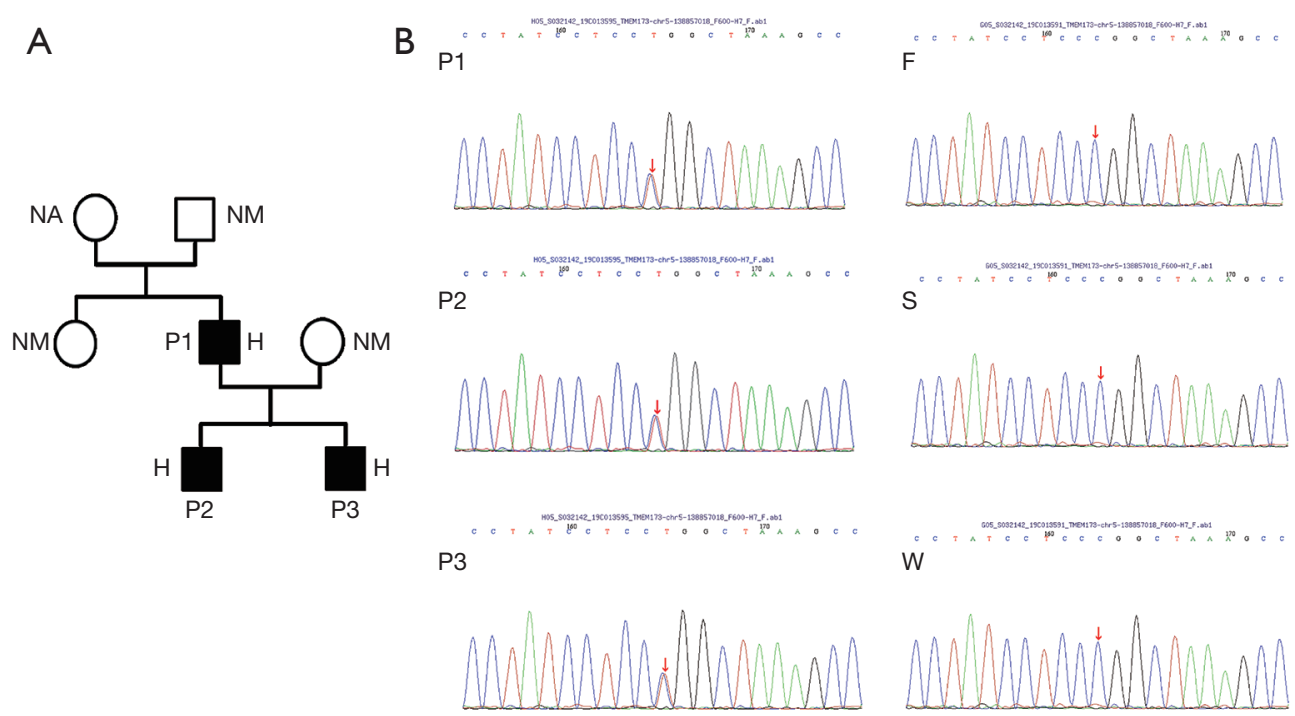

Figure 4 The genetic analysis of the three cases. (A) Family pedigrees of the 3 cases with the R281Q pathogenic variant in STING1. Solid symbols indicate affected relatives, open symbols indicate unaffected relatives, squares represent male persons, and circle represent female persons. H denotes heterozygous mutated gene, NA denotes not available, NM denotes nonmutated gene. (B) Whole-exome sequencing (WES) filtering was performed on patients and his relatives. $\mathrm{P}$ denotes the case; $\mathrm{F}$ demotes the father of case $1 ; \mathrm{S}$ denotes the sister of case 1 ; $W$ denotes the wife of case 1 .

from 1980 to November 2020, only 56 cases of SAVI have been reported to date (Table 1), including these three newly identified cases (Table 2). Among the published case reports, the age at presentation ranged from 1 day to 65 years. There were 30 (54\%) males, 22 (39\%) females, and 4 patients without sex information. The prominent clinical manifestations included ILD (39 cases, 70\%), developmental retardation (28 cases, 50\%), and skin lesions (43 cases, $78 \%$ ). In addition, 5 cases (9\%) had pulmonary hypertension, 4 cases exhibited hypothyroidism (8\%), and 9 cases (18\%) were asymptomatic in the respiratory system. All nine reported fatalities from SAVI were due to 


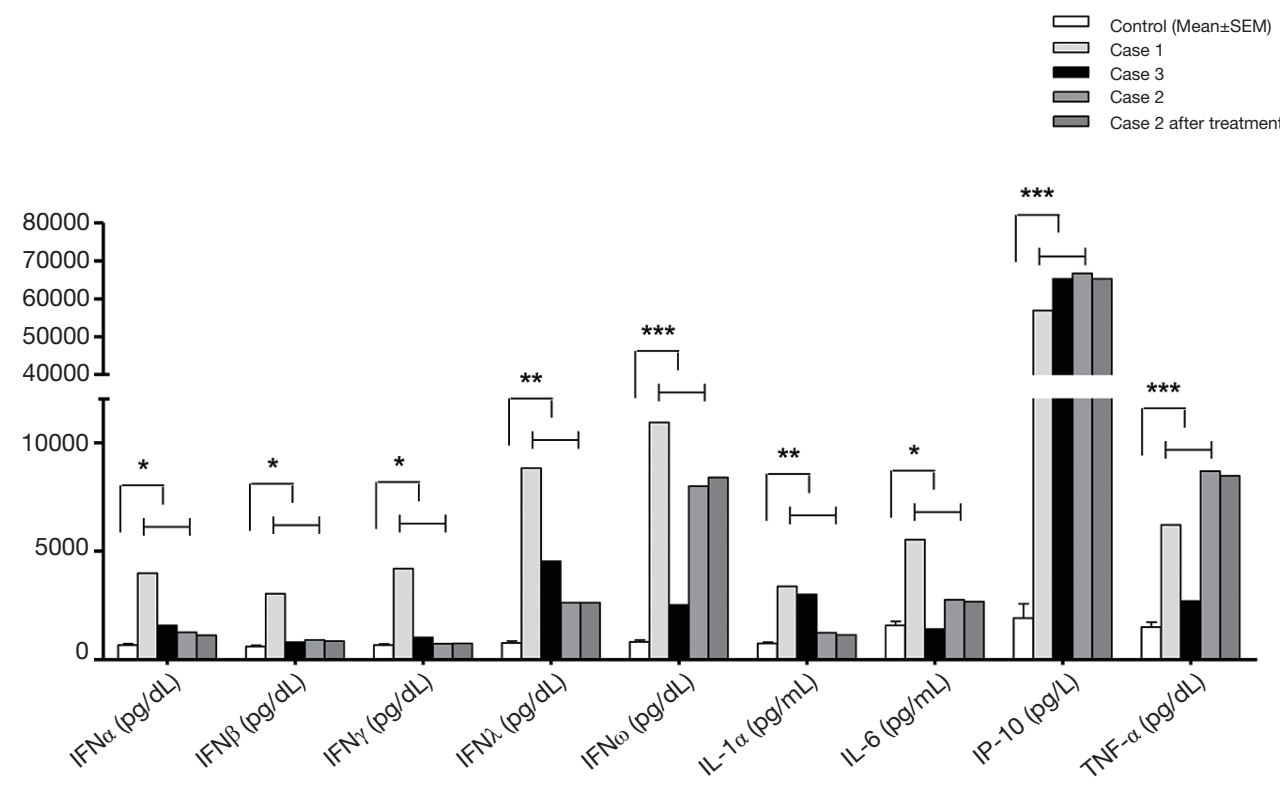

Figure 5 The activation of IFN proteins and inflammatory cytokines. The circulating IFN proteins and released inflammatory cytokines were measured via ELISA. Compared with controls ( $\mathrm{n}=10)$, serum levels of IFN proteins (IFN $\alpha$, IFN $\beta$, IFN $\gamma$, IFN $\lambda 1 / 2$ and IFN $\omega)$ and inflammatory cytokines (IL-1 $\alpha$, IL-6, IP-10 and TNF- $\alpha$ ) were significantly increased. After ruxolitinib treatment, no obvious reductions in IFN proteins (IFN $\alpha$, IFN $\beta$, IFN $\gamma$, IFN $\lambda 1 / 2$ and IFN $\omega$ ) and inflammatory cytokines (IL-1 $\alpha$, IL-6, IP-10 and TNF- $\alpha$ ) were observed in case 2 . The data were analyzed by the unpaired t test. The symbols $\left(^{*}\right),\left({ }^{* *}\right)$ and $\left(^{* * *}\right)$ indicate statistically significant changes at $\mathrm{P}<0.05, \mathrm{P}<0.01$, and $\mathrm{P}<0.001$, respectively.

pulmonary complications.

Either inherited or de novo, 12 types of activating STING1 pathogenic variants were identified in SAVI patients. The most common mutation was p.V155M (19 cases, 35\%). Only one case was found to have double mutations (p. S102P and p.F279L) (3). In addition to our three patients, there was another reported case with a heterozygous R281Q mutation with both ILD and evident skin lesions (6). Lin et al. recently reported 6 SAVI patients from 4 unrelated families with the recessive homozygous STING1 variant p.R281W (22). The severity and natural courses of the disease seem to be independent of the mutation type. For instance, a 25-year-old SAVI patient with R284G pathogenic variant had no lung involvement (6). However, another 9-month-old patient with the same location of pathogenic variant died shortly due to respiratory failure (13).

Information on treatment was available in 37 cases. Corticosteroids were used in 25 cases, and only 11 cases had limited evidence of improvement. Immunosuppressive agents were combined with corticosteroids in 16 cases without a significant additional effect. Recently, JAK inhibitors have been gradually brought to the forefront (2). Among the 20 patients receiving JAK inhibitors, the skin lesions and/or pulmonary impairment were variously improved in 10 cases. Additionally, 1 case received lung transplantation (3), and 1 case was prescribed salicylic acid. However, both exhibited poor responses (6).

\section{Discussion}

STING is a key player in the upstream regulation of the type I IFN response. Activated STING serves as an adaptor to induce the production and secretion of type I interferons (IFNs). Binding of IFNs to their receptors activates Janus kinases (JAKs) to upregulate the transcription of interferonstimulated genes. Specifically, JAK1 was involved in IFN beta and IFN alpha signaling. Therefore, the release of interferon leads to a positive feedback loop that further upregulates STING and the transcription of other proinflammatory cytokines. In patients with SAVI, constitutively activated STING leads to increased transcription of the type 1 interferon gene IFNB1, which encodes interferon- $\beta$. Binding of interferon- $\beta$ to its receptor activates JAKs, including 


\begin{tabular}{|c|c|c|c|c|c|c|c|c|c|c|}
\hline $\begin{array}{l}\text { Study, year of } \\
\text { publication }\end{array}$ & $\begin{array}{l}\text { Case number (sex } \\
\text { distribution), } \\
\text { age onset }\end{array}$ & $\begin{array}{l}\text { Lung involvement } \\
\text { [number of patients } \\
\text { afflicted] }\end{array}$ & $\begin{array}{l}\text { Developmental } \\
\text { retardation }\end{array}$ & $\begin{array}{l}\text { Cutaneous and other } \\
\text { presentations [number of } \\
\text { patients afflicted] }\end{array}$ & Inflammatory markers & Immune perturbation & $\begin{array}{l}\text { Autoantibody profile } \\
\text { [number of patients } \\
\text { afflicted] }\end{array}$ & $\begin{array}{l}\text { Gene variant } \\
\text { [number of } \\
\text { patients afflicted] }\end{array}$ & Treatment & Prognosis \\
\hline Liu et al., 2014 (1) & $\begin{array}{l}6(3 \mathrm{~F} / 3 \mathrm{M}), 1 \text { day to } \\
8 \mathrm{w}\end{array}$ & $\begin{array}{l}\text { ILD [5], paratracheal } \\
\text { adenopathy [6], } \\
\text { lung fibrosis [3] }\end{array}$ & Unknown & $\begin{array}{l}\text { Acral violaceous plaques [6], } \\
\text { skin nodules [6],nail dystrophy } \\
\text { or loss [6], nasal-septum } \\
\text { perforation [4] }\end{array}$ & Unknown & Unknown & $\begin{array}{l}\text { ANA+ [3], } \\
\text { Antiphospholipid } \\
\text { antibodies+ [5], c-ANCA+ [1] }\end{array}$ & $\begin{array}{l}\text { de novo p.N154S } \\
\text { [4], de novo } \\
\text { p.V155M [1], de } \\
\text { novo p.V147L [1] }\end{array}$ & $\begin{array}{l}\text { Glucocorticoid, DMARD, Biologic } \\
\text { agent }\end{array}$ & $\begin{array}{l}\text { No response or } \\
\text { incomplete response }\end{array}$ \\
\hline $\begin{array}{l}\text { Omoyinmi et al., } \\
2015(7)\end{array}$ & $1(\mathrm{M}), 1 \mathrm{w}$ & $\begin{array}{l}\text { ILD, pulmonary } \\
\text { hypertension }\end{array}$ & Unknown & Ulceration of the extremities & $\begin{array}{l}\text { Increased levels of } \\
\text { CRP, ESR }\end{array}$ & Unknown & $\begin{array}{l}\text { Anticardiolipin IgG+, lupus } \\
\text { anticoagulant+ }\end{array}$ & de novo p.V155M & $\begin{array}{l}\text { Corticosteroids, } \\
\text { cyclophosphamide, } \\
\text { azathioprine, methotrexate, } \\
\text { rituximab and infliximab }\end{array}$ & Deceased (at 16y) \\
\hline \multirow[t]{4}{*}{$\begin{array}{l}\text { Jeremiah et al., } \\
2014 \text { (8) }\end{array}$} & $\begin{array}{l}1 \text { (M), } 65 \text { y } \\
\text { (proband's grandpa) }\end{array}$ & Normal & Yes & Unknown & $\begin{array}{l}\text { Increased levels of } \\
\text { CRP, ESR }\end{array}$ & Unknown & ANA 1:340 & pV155M & Nil & Unknown \\
\hline & $\begin{array}{l}1 \text { (M), } 29 \mathrm{y} \\
\text { (proband's father) }\end{array}$ & ILD & Yes & Unknown & $\begin{array}{l}\text { Increased levels of } \\
\text { CRP, ESR }\end{array}$ & Unknown & ANA 1:640 & Inherited pV155M & Nil & Deceased \\
\hline & $\begin{array}{l}1 \text { (M), } 33 \text { y } \\
\text { (proband's uncle) }\end{array}$ & ILD & Yes & Unknown & $\begin{array}{l}\text { Increased levels of } \\
\text { CRP, ESR }\end{array}$ & Unknown & ANA 1:320 & Inherited pV155M & Nil & Unknown \\
\hline & $1(\mathrm{~F}), 4 \mathrm{y}$ (proband) & ILD & Yes & Unknown & $\begin{array}{l}\text { Increased levels of } \\
\text { CRP, ESR }\end{array}$ & Unknown & ANA 1:200-1:800 & Inherited pV155M & Nil & Unknown \\
\hline $\begin{array}{l}\text { Munoz, et al., } \\
2015 \text { (9) }\end{array}$ & 1 (M), $38 \mathrm{w}$ & ILD stable & Yes & $\begin{array}{l}\text { Skin necrosis, gangrene, } \\
\text { Nasal septum perforation }\end{array}$ & Normal & Unknown & ANCA 1:200-1:400 & de novo p.V147M & $\begin{array}{l}\text { Methyl|prednisolone,prednisone, } \\
\text { clopidogrel bisulfate, nifedipine }\end{array}$ & $\begin{array}{l}\text { Pulmonary feature } \\
\text { stable, skin lesions } \\
\text { worse }\end{array}$ \\
\hline \multirow[t]{3}{*}{$\begin{array}{l}\text { Picard, et al., } \\
2016 \text { (3) }\end{array}$} & 1 (M), $12 y$ & Pulmonary fibrosis & Yes & Telangiectasia and chilblains & $\begin{array}{l}\text { Increased levels of } \\
\text { CRP, ESR }\end{array}$ & $\begin{array}{l}\text { CD4+ lymphopenia, NK cell } \\
\text { decreased, } \\
\text { hypergammaglobulinemia } \\
\text { (IgG, IgA) }\end{array}$ & ANAt+ & de novo p.V155M & Nil & Unknown \\
\hline & $1(\mathrm{~F}), 20 \mathrm{y}$ & $\begin{array}{l}\text { Pulmonary fibrosis, } \\
\text { emphysema }\end{array}$ & Unknown & $\begin{array}{l}\text { Acral telangiectasia, atrophic } \\
\text { plaques, nail dystropy }\end{array}$ & Unknown & $\begin{array}{l}\text { CD4+ lymphopenia, NK cell } \\
\text { decreased, } \\
\text { hypergammaglobulinemia (IgG) }\end{array}$ & ANA,+ c-ANCA+ & de novo p.V155M & Lung transplantation & Deceased \\
\hline & $1(\mathrm{M}), 5 \mathrm{~m}$ & ILD & Yes & Telangiectasia and chilblains & $\begin{array}{l}\text { Increased levels of } \\
\text { CRP, ESR }\end{array}$ & $\begin{array}{l}\text { CD4+ and CD8+ Iymphopenia, } \\
\text { hypergammaglobulinemia (IgG) }\end{array}$ & Nil & de novo p.V155M & Nil & Unknown \\
\hline \multirow[t]{2}{*}{$\begin{array}{l}\text { Frémond et al., } \\
2016(4)\end{array}$} & $\begin{array}{l}1(\mathrm{M}), 12 \text { y (reported } \\
\text { by Picard, et al., } \\
\text { 2016) }\end{array}$ & Lung fibrosis & Yes & $\begin{array}{l}\text { Chilblains of the feet and left } \\
\text { ear }\end{array}$ & $\begin{array}{l}\text { Increased levels of } \\
\text { CRP, ESR }\end{array}$ & $\begin{array}{l}\text { CD4+ lymphopenia, NK cell } \\
\text { decreased, } \\
\text { hypergammaglobulinemia } \\
\text { (IgG, IgA) }\end{array}$ & ANAt+ & de novo p.V155M & $\begin{array}{l}\text { Hydroxychloroquine, steroids, } \\
\text { ruxolitinib }\end{array}$ & $\begin{array}{l}\text { Improved for lung } \\
\text { symptoms but not skin } \\
\text { lesions }\end{array}$ \\
\hline & $\begin{array}{l}1 \text { (F), neonate } \\
\text { (reported by } \\
\text { Jeremiah et al., } \\
\text { 2014) }\end{array}$ & ILD & Yes & $\begin{array}{l}\text { Occasional erythema of the } \\
\text { cheeks }\end{array}$ & $\begin{array}{l}\text { Increased levels of } \\
\text { CRP, ESR }\end{array}$ & Unknown & ANA 1:200-1:800 & Inherited pV155M & $\begin{array}{l}\text { Steroids, MMF, anti-CD20 } \\
\text { monoclonal antibodies, ruxolitinib }\end{array}$ & $\begin{array}{l}\text { Improvement of } \\
\text { interstitial pneumonitis }\end{array}$ \\
\hline $\begin{array}{l}\text { Seo, et al., } \\
2017 \text { (10) }\end{array}$ & $1(M), 9 y$ & $\begin{array}{l}\text { Obliterative } \\
\text { bronchiolitis }\end{array}$ & Unknown & $\begin{array}{l}\text { Perforated nasal septum, } \\
\text { gangrenous lesions }\end{array}$ & $\begin{array}{l}\text { Increased levels of } \\
\text { CRP, ESR }\end{array}$ & $\begin{array}{l}\text { CD4+ lymphopenia, } \\
\text { monocytes increased }\end{array}$ & Unknown & $\begin{array}{l}\text { de novo p.S102P, } \\
\text { de novo p.F279L }\end{array}$ & Tofacitinib & $\begin{array}{l}\text { Only skin lesions } \\
\text { improved }\end{array}$ \\
\hline $\begin{array}{l}\text { Clarke et al., } \\
2016 \text { (11) }\end{array}$ & $1(\mathrm{M}), 5 \mathrm{w}$ & ILD & Unknown & $\begin{array}{l}\text { Maculo } \\
\text { papular, } \\
\text { erythematous lesions }\end{array}$ & $\begin{array}{l}\text { Increased levels of } \\
\text { CRP }\end{array}$ & $\lg G$ and $\lg A$ increased & SSAt & de novo p.V155M & $\begin{array}{l}\text { Methylprednisolone and } \\
\text { immunoglobulin }\end{array}$ & Improved \\
\hline
\end{tabular}

Table 1 (continued) 


\begin{tabular}{|c|c|c|c|c|c|c|c|c|c|c|}
\hline $\begin{array}{l}\text { Study, year of } \\
\text { publication }\end{array}$ & $\begin{array}{l}\text { Case number (sex } \\
\text { distribution), } \\
\text { age onset }\end{array}$ & $\begin{array}{l}\text { Lung involvement } \\
\text { [number of patients } \\
\text { afflicted] }\end{array}$ & $\begin{array}{l}\text { Developmental } \\
\text { retardation }\end{array}$ & $\begin{array}{l}\text { Cutaneous and other } \\
\text { presentations [number of } \\
\text { patients afflicted] }\end{array}$ & Inflammatory markers & Immune perturbation & $\begin{array}{l}\text { Autoantibody profile } \\
\text { [number of patients } \\
\text { afflicted] }\end{array}$ & $\begin{array}{l}\text { Gene variant } \\
\text { [number of } \\
\text { patients afflicted] }\end{array}$ & Treatment & Prognosis \\
\hline \multirow[t]{3}{*}{$\begin{array}{l}\text { Melki et al., } \\
2017 \text { (6) }\end{array}$} & $1(\mathrm{~F}), 25 \mathrm{y}$ & Normal & Yes & $\begin{array}{l}\text { Livido Acrocyanosis, palatal/ } \\
\text { nasal septum necrosis }\end{array}$ & Normal & Unknown & ANA $1: 160$, ANCA- & de novo p.R284G & Nil & Unknown \\
\hline & $1(\mathrm{~F}), 3 \mathrm{~m}$ & ILD & Yes & Early-onset malar rash & $\begin{array}{l}\text { Increased of } \\
\text { inflammatory markers }\end{array}$ & Unknown & Nil & de novo p.R281Q & $\begin{array}{l}\text { Steroids, methotrexate, anti- } \\
\text { TNF } \alpha\end{array}$ & No efficacy \\
\hline & 1 (M), $15 \mathrm{y}$ & Normal & No & $\begin{array}{l}\text { Livedo and dark purple } \\
\text { marbling on the limbs, } \\
\text { painful acralerosions on the } \\
\text { ear helices and digits }\end{array}$ & Normal & Normal & Nil & de novo p.C206Y & $\begin{array}{l}\text { Nifedipin, pentoxyfillin, acetyl, } \\
\text { salicylic acid }\end{array}$ & $\begin{array}{l}\text { Limited efficacy for skin } \\
\text { lesions }\end{array}$ \\
\hline $\begin{array}{l}\text { Konno } \\
\text { et al., } 2018 \text { (13) }\end{array}$ & $1(\mathrm{M}), 9 \mathrm{~m}$ & Normal & No & No & $\begin{array}{l}\text { Increased level of } \\
\text { IL-6 }\end{array}$ & Unknown & Unknown & de novo p.R284S & Nil & Deceased \\
\hline $\begin{array}{l}\text { Sanchez et al., } \\
2018 \text { (5) }\end{array}$ & 4 (unknown), 0-18 y & ILD [4] & Yes & $\begin{array}{l}\text { Cutaneous vasculitis [4], } \\
\text { ulcers [3], }\end{array}$ & $\begin{array}{l}\text { Increased levels of } \\
\text { CRP, ESR }\end{array}$ & Unknown & Unknown & de novo p.N154S & Baricitinib & $\begin{array}{l}\text { Improved for lung } \\
\text { function }\end{array}$ \\
\hline $\begin{array}{l}\text { Yu et al., } \\
2018(14)\end{array}$ & $1(\mathrm{M}), 2 \mathrm{~m}$ & ILD & Yes & $\begin{array}{l}\text { Cold-induced violaceous } \\
\text { papules or plaques on ears }\end{array}$ & $\begin{array}{l}\text { Increased levels of } \\
\text { CRP, ESR, IL-6 and } \\
\text { TNF- } \alpha\end{array}$ & $\begin{array}{l}\text { Hypergammaglobulinemia (IgG, } \\
\operatorname{lgA} A\end{array}$ & $\mathrm{RF}_{+}$ & de novo p.V155M & Tofacitinib & $\begin{array}{l}\text { Improved for lung } \\
\text { function }\end{array}$ \\
\hline $\begin{array}{l}\text { Shoman et al., } 2019 \\
\text { (15) }\end{array}$ & $1(\mathrm{~F}), 5 \mathrm{y}$ & ILD & Yes & $\begin{array}{l}\text { Atrophic scars, destructed } \\
\text { nasal septum, destructed } \\
\text { nails }\end{array}$ & $\begin{array}{l}\text { Increased levels of } \\
\text { CRP, ESR }\end{array}$ & Unknown & ANA+ & de novo p.N154S & Methotrexate, prednisolone & $\begin{array}{l}\text { Improved for lung } \\
\text { disease and cutaneous } \\
\text { lesions }\end{array}$ \\
\hline \multirow[t]{3}{*}{ Volpi et al., 2019 (2) } & $1(\mathrm{~F}), 8 \mathrm{~m}$ & ILD & Yes & $\begin{array}{l}\text { Maculopapular lesions } \\
\text { evolving to ulcers }\end{array}$ & $\begin{array}{l}\text { Increased levels of } \\
\text { CRP, ESR }\end{array}$ & Normal & ANA,+ p-ANCA+, PR3+ & de novo p.V155M & Ruxolitinib & $\begin{array}{l}\text { Improved for lung } \\
\text { disease and cutaneous } \\
\text { lesions }\end{array}$ \\
\hline & $1(\mathrm{~F}), 3 \mathrm{~m}$ & ILD & Yes & Malar rash & $\begin{array}{l}\text { Increased level of } \\
\text { ESR }\end{array}$ & Normal & $\begin{array}{l}\text { CANCA+, anti-cardiolipin+, } \\
\text { B2GLG+ }\end{array}$ & de novo p.R281Q & Ruxolitinib & $\begin{array}{l}\text { Skin lesions and ILD } \\
\text { worsened }\end{array}$ \\
\hline & $1(\mathrm{~F}), 3 \mathrm{~m}$ & ILD & Yes & Erythematosus vesicular rash & $\begin{array}{l}\text { Increased levels of } \\
\text { CRP, ESR }\end{array}$ & Normal & $\begin{array}{l}\text { cANCA+, PL+, } \\
\text { anti-cardiolipin+, PT+ }\end{array}$ & de novo p.N154S & Ruxolitinib & $\begin{array}{l}\text { Transient improvement } \\
\text { for lung disease and } \\
\text { skin lesions, and then } \\
\text { lapse of ILD }\end{array}$ \\
\hline $\begin{array}{l}\text { Balci et al., } \\
2019 \text { (16) }\end{array}$ & $1(\mathrm{M}), 6 \mathrm{~m}$ & ILD & No & $\begin{array}{l}\text { Purplish red non-indurating } \\
\text { plaques and pustular lesions }\end{array}$ & $\begin{array}{l}\text { Increased levels of } \\
\text { CRP, ESR }\end{array}$ & Normal & Nil & de novo p.N154S & Ruxolitinib switch to baricitinib & $\begin{array}{l}\text { Greatly improved for } \\
\text { lung and cutaneous } \\
\text { lesions }\end{array}$ \\
\hline \multirow[t]{2}{*}{ Tang et al., 2020 (17) } & $1(\mathrm{~F}), 4 \mathrm{~m}$ & ILD & Yes & No & $\begin{array}{l}\text { Increased level of } \\
\text { ESR }\end{array}$ & $\begin{array}{l}\text { Hypergammaglobulinemia IgG } \\
\text { and decreased NK cells, } \\
\text { decreased CD3+ lymphocytes } \\
\text { and } C D 3+C D 8+\text { lymphocytes }\end{array}$ & $\begin{array}{l}\text { ANCA+ (25 } \mathrm{m} \text { after the } \\
\text { onset), ANA 1:80 }\end{array}$ & de novo p.V155M & Corticosteroids, IVIG & $\begin{array}{l}\text { Improved for lung and } \\
\text { cutaneous lesions }\end{array}$ \\
\hline & $1(\mathrm{~F}), 3 \mathrm{~m}$ & ILD & Yes & $\begin{array}{l}\text { Angiotelectasis, chilblain } \\
\text { lesions }\end{array}$ & Normal & $\begin{array}{l}\text { Hypergammaglobulinemia IgG, } \\
\text { decreased NK cells, } \\
\text { decreased CD3+ lymphocytes } \\
\text { and CD3+CD8+ lymphocytes }\end{array}$ & Unknown & de novo p.V155M & Corticosteroids, IVIG, tofacitinib & Deceased \\
\hline
\end{tabular}

Table 1 (continued) 


\begin{tabular}{|c|c|c|c|c|c|c|c|c|c|c|}
\hline $\begin{array}{l}\text { Study, year of } \\
\text { publication }\end{array}$ & $\begin{array}{l}\text { Case number (sex } \\
\text { distribution), } \\
\text { age onset }\end{array}$ & $\begin{array}{l}\text { Lung involvement } \\
\text { [number of } \\
\text { patients afflicted] }\end{array}$ & $\begin{array}{l}\text { Developmental } \\
\text { retardation }\end{array}$ & $\begin{array}{l}\text { Cutaneous and other } \\
\text { presentations [number of } \\
\text { patients afflicted] }\end{array}$ & Inflammatory markers & Immune perturbation & $\begin{array}{l}\text { Autoantibody profile } \\
\text { [number of patients } \\
\text { afflicted] }\end{array}$ & $\begin{array}{l}\text { Gene variant } \\
\text { [number of } \\
\text { patients afflicted] }\end{array}$ & Treatment & Prognosis \\
\hline & 1 (M), $54 \mathrm{~m}$ & ILD & No & $\begin{array}{l}\text { Angiotelectasis, } \\
\text { erythematous, purpuric or } \\
\text { scaling rashes }\end{array}$ & $\begin{array}{l}\text { Increased levels of } \\
\text { CRP, ESR }\end{array}$ & $\begin{array}{l}\text { Hypergammaglobulinemia lgG } \\
\text { and decreased NK cells }\end{array}$ & $\mathrm{RF}+, \mathrm{CCP}+$ & de novo p.V155M & $\begin{array}{l}\text { Corticosteroids, IVIG, CTX, } \\
\text { tofacitinib }\end{array}$ & $\begin{array}{l}\text { Respiratory symptoms } \\
\text { improved but rashes } \\
\text { worsened }\end{array}$ \\
\hline \multirow[t]{5}{*}{$\begin{array}{l}\text { Keskitalo et al., } \\
2019 \text { (18) }\end{array}$} & 1 (M), birth & Normal & Unknown & $\begin{array}{l}\text { Livedo reticularis, skin } \\
\text { vasculitis, nasal septal } \\
\text { perforation, facial erythema, } \\
\text { UV sensitivity, alopecia, skin } \\
\text { infections }\end{array}$ & Unknown & $\begin{array}{l}\text { Decreased CD19+ B cells and } \\
\text { NK cells, increased lgE }\end{array}$ & Negative & Inherited p. G207E & $\begin{array}{l}\text { Prednisolone combined with } \\
\text { azathioprine or methotrexate, } \\
\text { cyclosporine, baricitinib }\end{array}$ & $\begin{array}{l}\text { Improved for alopecia } \\
\text { and over-all well being }\end{array}$ \\
\hline & $1(F)$, birth & Normal & Unknown & $\begin{array}{l}\text { Livedo reticularis, facial } \\
\text { erythema, UV sensitivity, } \\
\text { alopecia, skin infections, } \\
\text { hypothyroid }\end{array}$ & Unknown & Increased IgE & Negative & Inherited p. G207E & Unknown & Unknown \\
\hline & $1(\mathrm{~F})$, birth & Normal & Unknown & $\begin{array}{l}\text { Livedo reticularis, nasal septal } \\
\text { perforation, facial erythema, } \\
\text { UV sensitivity, alopecia, } \\
\text { autoimmune thyroiditis }\end{array}$ & Unknown & Normal & Negative & Inherited p. G207E & Unknown & Unknown \\
\hline & $1(F)$, birth & Normal & Unknown & $\begin{array}{l}\text { Livedo reticularis, facial } \\
\text { erythema, UV sensitivity, skin } \\
\text { infections, periodontitis }\end{array}$ & Unknown & Increased lgE & Negative & Inherited p. G207E & Unknown & Unknown \\
\hline & $1(\mathrm{~F})$, birth & Normal & Unknown & $\begin{array}{l}\text { Livedo reticularis, UV } \\
\text { sensitivity, alopcia, } \\
\text { autoimmune thyroiditis, } \\
\text { periodontitis }\end{array}$ & Unknown & Increased lgE & Negative & Inherited p. G207E & $\begin{array}{l}\text { Radioiodine treatment, thyroid } \\
\text { hormone substitution, calcium } \\
\text { and vitamin D- replacement } \\
\text { therapy }\end{array}$ & Improved for alopecia \\
\hline \multirow[t]{2}{*}{$\begin{array}{l}\text { Cao et al., } \\
2019 \text { (19) }\end{array}$} & $1(\mathrm{M}), 2 \mathrm{~m}$ & ILD & Yes & Rash & $\begin{array}{l}\text { Increased levels of } \\
\text { CRP, ESR }\end{array}$ & $\begin{array}{l}\text { Increased IgE, } \\
\text { hypergammaglobulinemia IgA, }\end{array}$ & Negative & de novo p.V155M & Unknown & Unknown \\
\hline & $1(\mathrm{M}), 1 \mathrm{~m}$ & ILD & Yes & Telangiectatic skin lesions & $\begin{array}{l}\text { Increased levels of } \\
\text { CRP, ESR }\end{array}$ & $\begin{array}{l}\text { Increased lgE, } \\
\text { hypergammaglobulinemia IgA, } \\
\text { decreased } \operatorname{lgG} \text { and } \operatorname{lgM}\end{array}$ & $\mathrm{CCP}+$ & de novo p.N154S & Unknown & Unknown \\
\hline $\begin{array}{l}\text { Clarke } \\
\text { et al., } 2020 \text { (20) }\end{array}$ & $1(F)$, neonate & ILD & Yes & No & $\begin{array}{l}\text { Increased level of } \\
\text { CRP }\end{array}$ & $\begin{array}{l}\text { Hypergammaglobulinemia (IgG, } \\
\text { IgA and IgM) }\end{array}$ & Anti-smooth muscle+ & Inherited p.V155M & $\begin{array}{l}\text { Methylprednisolone, IVIG, } \\
\text { baricitinib }\end{array}$ & $\begin{array}{l}\text { Improved for lung } \\
\text { diseases }\end{array}$ \\
\hline
\end{tabular}




\begin{tabular}{|c|c|c|c|c|c|c|c|c|c|c|}
\hline $\begin{array}{l}\text { Study, year of } \\
\text { publication }\end{array}$ & $\begin{array}{l}\text { Case number (sex } \\
\text { distribution), } \\
\text { age onset }\end{array}$ & $\begin{array}{l}\text { Lung involvement } \\
\text { [number of } \\
\text { patients afflicted] }\end{array}$ & $\begin{array}{l}\text { Developmental } \\
\text { retardation }\end{array}$ & $\begin{array}{l}\text { Cutaneous and other } \\
\text { presentations [number of } \\
\text { patients afflicted] }\end{array}$ & Inflammatory markers & Immune perturbation & $\begin{array}{l}\text { Autoantibody profile } \\
\text { [number of patients } \\
\text { afflicted] }\end{array}$ & $\begin{array}{l}\text { Gene variant } \\
\text { [number of } \\
\text { patients afflicted] }\end{array}$ & Treatment & Prognosis \\
\hline & $1(F), 22 \mathrm{~m}$ & ILD & Unknown & Rash & Normal & $\begin{array}{l}\text { Hypergammaglobulinemia (IgG, } \\
\text { IgA and IgM) }\end{array}$ & ANA $1: 320$, RF+ & Inherited p.V155M & $\begin{array}{l}\text { Oral prednisolone, rituximab, } \\
\text { baricitinib }\end{array}$ & $\begin{array}{l}\text { Improved for lung } \\
\text { diseases }\end{array}$ \\
\hline & $1(F), 16 y$ & ILD & Unknown & Unknown & Normal & Hypergammaglobulinemia IgA & $\begin{array}{l}\text { ANA 1:80/negative, } \\
\text { Anti-dsDNA+, RF+ }\end{array}$ & de novo p.V155M & Oral and intravenous steroids & Deceased \\
\hline $\begin{array}{l}\text { Abid } \\
\text { et al., } 2020 \text { (21) }\end{array}$ & $1(M), 4 \mathrm{w}$ & ILD & Yes & $\begin{array}{l}\text { Blisters, ulcers, digital } \\
\text { ischemia, nephroticrange } \\
\text { proteinuria }\end{array}$ & $\begin{array}{l}\text { Increased levels of } \\
\text { inflammatory markers }\end{array}$ & Unknown & Unknown & de novo p.V147L & Ruxolitinib, methylprednisolone & $\begin{array}{l}\text { Transient improvement } \\
\text { for skin lesions and lung } \\
\text { disease }\end{array}$ \\
\hline \multirow[t]{6}{*}{ Lin et al., 2020 (22) } & $\begin{array}{l}1(\mathrm{M}), 4 \mathrm{~W}, \\
\text { proband } \mathrm{A}\end{array}$ & ILD & Yes & $\begin{array}{l}\text { Maculopapular } \\
\text { violaceous rash with a } \\
\text { livedoid appearance }\end{array}$ & Unknown & Unknown & Unknown & p.R281W & Steroids, tofacitinib & Deceased \\
\hline & $\begin{array}{l}1(\mathrm{M}), 18 \mathrm{~m} \\
\text { (proband A's } \\
\text { brother) }\end{array}$ & Chronic cough & Yes & Unknown & Unknown & Unknown & Unknown & Unknown & Unknown & Deceased \\
\hline & $\begin{array}{l}1(\mathrm{M}), 3 \mathrm{~m}, \text { proband } \\
\mathrm{B}\end{array}$ & ILD & Unknown & Erythematous rash & Unknown & Unknown & Unknown & p.R281W & Ruxolitinib & Unknown \\
\hline & $\begin{array}{l}1 \text { (M), } 6 \mathrm{~m} \text { (proband } \\
\text { B's brother) }\end{array}$ & Unknown & Unknown & Unknown & Unknown & Unknown & Unknown & p.R281W & Steroids, baricitinib & Unknown \\
\hline & $1(F), 2 m$ & $\begin{array}{l}\text { ILD, recurrent lung } \\
\text { infections and } \\
\text { pulmonary } \\
\text { hypertension }\end{array}$ & Unknown & $\begin{array}{l}\text { Erythematous rash and } \\
\text { polyarthritis }\end{array}$ & Unknown & Unknown & Unknown & p.R281W & Baricitinib & $\begin{array}{l}\text { Clinical improvement, } \\
\text { still oxygen dependence }\end{array}$ \\
\hline & $1(\mathrm{M}), 8 \mathrm{~m}$ & Unknown & Yes & $\begin{array}{l}\text { Intermittent vasculitic rashes } \\
\text { and polyarthritis }\end{array}$ & Unknown & Unknown & Unknown & p.R281W & Unknown & Unknown \\
\hline
\end{tabular}

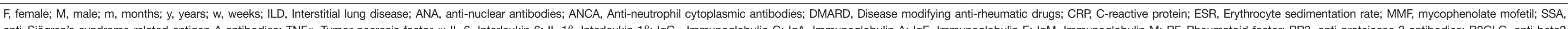

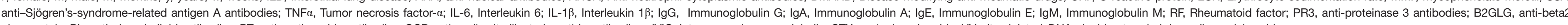


Table 2 Clinical characteristics of the 4 patients with R281Q pathogenic variant reported in the literature and this study

\begin{tabular}{|c|c|c|c|c|c|c|c|c|c|c|c|}
\hline Case & $\begin{array}{l}\text { Age } \\
\text { onset }\end{array}$ & $\begin{array}{l}\text { Current } \\
\text { age }\end{array}$ & Sex & Cutaneous & Lung & Other systems & $\begin{array}{l}\text { Growth } \\
\text { retardation }\end{array}$ & $\begin{array}{l}\text { Frequent } \\
\text { infection }\end{array}$ & $\begin{array}{c}\text { inflammatory } \\
\text { markers }\end{array}$ & $\begin{array}{l}\text { Antibody } \\
\text { status }\end{array}$ & $\begin{array}{l}\text { Treatments } \\
\text { and prognosis }\end{array}$ \\
\hline $\begin{array}{l}\text { Previous } \\
\text { reported } \\
\text { case (4) }\end{array}$ & $3 \mathrm{~m}$ & $7 y$ & Female & $\begin{array}{l}\text { Early-onset } \\
\text { malar rash }\end{array}$ & ILD, PAH & $\begin{array}{c}\text { Necrotizing } \\
\text { granulomatous } \\
\text { hepatitis }\end{array}$ & Yes & $\begin{array}{c}\text { Pneumonia } \\
\text { and skin } \\
\text { infections }\end{array}$ & Elevated & Nil & $\begin{array}{l}\text { Limited } \\
\text { efficacy with } \\
\text { steroids, } \\
\text { methotrexate, } \\
\text { and anti-TNF- } \alpha\end{array}$ \\
\hline Case 1 & $19 y$ & $37 y$ & Male & $\begin{array}{l}\text { Clubbing } \\
\text { fingers, nail, } \\
\text { dystrophy of } \\
\text { toes }\end{array}$ & ILD, PAH & No & No & No & Elevated & $\begin{array}{l}\text { Multiple } \\
\text { positive } \\
\text { antibodies }\end{array}$ & $\begin{array}{l}\text { Died } 4 \text { months } \\
\text { after ruxolitinib } \\
\text { treatment }\end{array}$ \\
\hline Case 3 & $2 y$ & $6 y$ & Male & Angiotelectasis & ILD & No & Yes & No & Elevated & ANA (+) & Nil \\
\hline
\end{tabular}

m, months; y, years; ILD, Interstitial lung disease; PAH, pulmonary artery hypertension; ANA, anti-nuclear antibodies; TNF- $\alpha$, tumor necrosis factor- $\alpha$.

JAK1 and tyrosine kinase 2 (TYK2), which subsequently results in receptor phosphorylation. This process allows the binding of the DNA-binding proteins signal transducers and activators of transcription 1 (STAT1) and 2 (STAT2) to the receptor, whereupon they become phosphorylated (P). Phosphorylation allows them to dimerize, and the dimer translocates to the nucleus, where it upregulates transcription of interferon-response genes, including interferon regulatory factor 7-dependent transcription of type 1 interferon genes. The synthesis and release of interferons and their binding to interferon receptors further upregulate STING and the transcription of other proinflammatory cytokine genes in a positive feedback loop. In our three cases, p.R281Q pathogenic variant in STING1 activated IFN protein upregulation, which may further stimulate the release of inflammatory cytokines, such as IL-6, IL-1 $\alpha$, IP-10 and TNF- $\alpha$. In addition, JAKs itself can directly act on downstream inflammatory effectors of STING.

The observed GOF of the STING autoactivating and disease-causing mutations was recently "explained" by 2 structural models (22). One model is that the binding of cGAMP to the STING dimer leads to a $180^{\circ}$ rotation of the ligand-binding domain and further releases inhibitors, such as p. N154S and p.V155M. Another model refers to mutations in the polymer interface (R281 and R284 mutant) directly resulting in inability of inhibitor binding, which causes STING autoactivation. In addition, as the p.Arg281 and p.Arg284 lie on the surface of the protein outside of the STING dimerization domain (6), these two mutations induce a conformational change in STING, plausibly enabling ERGolgi trafficking and TBK1/IRF3 phosphorylation in the absence of cGAMP binding. It has been reported that a JAK inhibitor (tofacitinib) failed to inhibit dsDNA-triggered, STING-dependent IRF3 phosphorylation in reconstituted cells expressing R284S in vitro (13). Volpi et al. reported worsening of interstitial disease after transient amelioration in a patient with c.842G>A p. Arg281Gln mutation (2). For the two patients receiving JAK inhibitors in Lin et al.'s report (22), one patient exhibited clinical improvement with continued oxygen dependence, and the other patient died of respiratory failure disease despite steroid therapy and short-term treatment with the JAK inhibitor tofacitinib. The above studies may provide some explanations for the observed unsatisfactory response of JAK inhibitors for patients with 281 or 284 mutants; however, there is no robust evidence to support the linkage between the treatment response and mutation type since too few patients have been reported in the literature. It has been reported recently that the stimulation of AMPK activity releases ULK1-mediated phosphorylation of STING, which inhibits STING's ability to phosphorylate IRF3. Therefore, ULK1 regulators may be an alternative therapeutic option for 
STING-induced inflammatory diseases (13), which should be further studied.

In our case report, the two patients seemed to exhibit poor response to JAK inhibitors ruxolitinib and IFN proteins, and the circulating inflammatory cytokines did not decrease with treatment in case 2. One possible explanation is that the low dose of ruxolitinib may not be sufficient to modulate the expression of the IFN signature, and it cannot be excluded that the non-responsiveness to ruxolitinib might be attributed to a dosing problem. It should also be noted that the measurement of IFN protein with ELISAs may not be sensitive or reliable enough to detect the changes in these biomarkers. In addition, disease severity may also play a role, and the reported variable clinical response to JAK inhibitor therapy may be related to different disease statuses. The limited effects of ruxolitinib, especially in case 1, may be due to advanced ILD/heart failure beyond any therapeutic measures, and an earlier introduction of ruxolitinib may still improve or limit the evolution of SAVI. Further investigation should focus on the early intervention effect of JAK inhibitors in patients with SAVI.

In conclusion, some crucial issues of SAVI require further exploration. Although it has been hypothesized that STING gain-of-function pathogenic variants cause disease through type I interferon signaling, the extent to which type I IFNs and IFN-induced genes promote disease manifestations or simply provide a surrogate signature of nucleic acid sensor activation remains controversial. Moreover, the therapeutic effect of JAK inhibitors on different phenotypes and disease statuses of SAVI warrants further investigation.

\section{Acknowledgments}

Funding: National Science Foundation for Young Scientists of China: (Grant No. 81700001).

\section{Footnote}

Reporting Checklist: The authors have completed the CARE reporting checklist. Available at http://dx.doi.org/10.21037/ atm-20-6198

Conflicts of Interest: All authors have completed the ICMJE uniform disclosure form (available at http://dx.doi. org/10.21037/atm-20-6198). The authors have no conflicts of interest to declare.

Ethical Statement: The authors are accountable for all aspects of the work in ensuring that questions related to the accuracy or integrity of any part of the work are appropriately investigated and resolved. All procedures performed in studies involving human participants were in accordance with the ethical standards of our institutional ethics committee and with the Helsinki Declaration (as revised in 2013). Written informed consent was obtained from the patient.

Open Access Statement: This is an Open Access article distributed in accordance with the Creative Commons Attribution-NonCommercial-NoDerivs 4.0 International License (CC BY-NC-ND 4.0), which permits the noncommercial replication and distribution of the article with the strict proviso that no changes or edits are made and the original work is properly cited (including links to both the formal publication through the relevant DOI and the license). See: https://creativecommons.org/licenses/by-nc-nd/4.0/.

\section{References}

1. Liu Y, Jesus AA, Marrero B, et al. Activated STING in a vascular and pulmonary syndrome. $\mathrm{N}$ Engl $\mathrm{J}$ Med 2014:371:507-18.

2. Volpi S, Insalaco A, Caorsi R, et al. Efficacy and Adverse Events During Janus Kinase Inhibitor Treatment of SAVI Syndrome. J Clin Immunol 2019;39:476-85.

3. Picard C, Thouvenin G, Kannengiesser C, et al. Severe Pulmonary Fibrosis as the First Manifestation of Interferonopathy (TMEM173 Mutation). Chest 2016;150:e65-71.

4. Fremond ML, Rodero MP, Jeremiah N, et al. Efficacy of the Janus kinase 1/2 inhibitor ruxolitinib in the treatment of vasculopathy associated with TMEM173activating mutations in 3 children. J Allergy Clin Immunol 2016;138:1752-5.

5. Sanchez GAM, Reinhardt A, Ramsey S, et al. JAK1/2 inhibition with baricitinib in the treatment of autoinflammatory interferonopathies. J Clin Invest 2018;128:3041-52.

6. Melki I, Rose Y, Uggenti C, et al. Disease-associated mutations identify a novel region in human STING necessary for the control of type I interferon signaling. J Allergy Clin Immunol 2017;140:543-52.e5.

7. Omoyinmi E, Melo Gomes S, Nanthapisal S, et al. Stimulator of interferon genes-associated vasculitis of infancy. Arthritis Rheumatol 2015;67:808.

8. Jeremiah N, Neven B, Gentili M, et al. Inherited STING- 
activating mutation underlies a familial inflammatory syndrome with lupus-like manifestations. J Clin Invest 2014;124:5516-20.

9. Munoz J, Rodière M, Jeremiah N, et al. Stimulator of Interferon Genes-Associated Vasculopathy With Onset in Infancy: A Mimic of Childhood Granulomatosis With Polyangiitis. JAMA Dermatol 2015;151:872-7.

10. Seo J, Kang JA, Suh DI, et al. Tofacitinib relieves symptoms of stimulator of interferon genes (STING)associated vasculopathy with onset in infancy caused by 2 de novo variants in TMEM173. J Allergy Clin Immunol 2017;139:1396-9.e12.

11. Clarke SL, Pellowe EJ, de Jesus AA, et al. Interstitial Lung Disease Caused by STING-associated Vasculopathy with Onset in Infancy. Am J Respir Crit Care Med 2016;194:639-42.

12. Saldanha RG, Balka KR, Davidson S, et al. A Mutation Outside the Dimerization Domain Causing Atypical STING-Associated Vasculopathy With Onset in Infancy. Front Immunol 2018;9:1535.

13. Konno H, Chinn IK, Hong D, et al. Pro-inflammation Associated with a Gain-of-Function Mutation (R284S) in the Innate Immune Sensor STING. Cell Rep 2018;23:1112-23.

14. Yu ZX, Zhong LQ, Song HM, et al. Stimulator of interferon genes-associated vasculopathy with onset in infancy: first case report in China. Zhonghua Er Ke Za Zhi 2018;56:179-85.

15. Shoman W, El Chazli Y, ElSawy I, et al. First Egyptian

Cite this article as: Wang Y, Wang F, Zhang X. STINGassociated vasculopathy with onset in infancy: a familial case series report and literature review. Ann Transl Med 2021;9(2):176. doi: 10.21037/atm-20-6198 patient with STING-associated vasculopathy with onset in infancy. Scand J Rheumatol 2019;48:338-9.

16. Balci S, Ekinci RMK, de Jesus AA, et al. Baricitinib experience on STING-associated vasculopathy with onset in infancy: A representative case from Turkey. Clin Immunol 2020;212:108273.

17. Tang X, Xu H, Zhou C, et al. STING-Associated Vasculopathy with Onset in Infancy in Three Children with New Clinical Aspect and Unsatisfactory Therapeutic Responses to Tofacitinib. J Clin Immunol 2020;40:114-22.

18. Keskitalo S, Haapaniemi E, Einarsdottir E, et al. Novel TMEM173 Mutation and the Role of Disease Modifying Alleles. Front Immunol 2019;10:2770.

19. Cao Y, Jiang LP. The Challenge of Diagnosing SAVI: Case Studies. Pediatr Allergy Immunol Pulmonol 2019; 32:167-72.

20. Clarke SLN, Robertson L, Rice GI, et al. Type 1 interferonopathy presenting as juvenile idiopathic arthritis with interstitial lung disease: report of a new phenotype. Pediatr Rheumatol Online J 2020;18:37.

21. Abid Q, Best Rocha A, Larsen CP, et al. APOL1Associated Collapsing Focal Segmental Glomerulosclerosis in a Patient With Stimulator of Interferon Genes (STING)-Associated Vasculopathy With Onset in Infancy (SAVI). Am J Kidney Dis 2020;75:287-90.

22. Lin B, Berard R, Al Rasheed A, et al. A novel STING1 variant causes a recessive form of STING-associated vasculopathy with onset in infancy (SAVI). J Allergy Clin Immunol 2020;146:1204-8.e6. 